This paper sets out to urge the quantification of adverse environmental impact on astronomy (AEI). Up to the present AEI has been treated anecdotally. This is not a criticism - the demonstrations have been compelling, particularly so in the case of light pollution. However, we must now move from demonstration to effective action. That effective action will impact the legitimate activities of others. Therefore astronomy will have to present evidence capable of convincing not just those sympathetic to the astronomical community, but the community in general that astronomy has a legitimate grievance and that the redress being sought will be effective in improving astronomical observing condition.

In order to be convincing we must quantify. We must be able to state, for example, what background levels of light pollution are, what the normal range of the level is and how the level is changing from year to year. All claimed adverse impact should have some measure to express the real nature of the impact. Only when such measures are in place can we argue for legal protection.

The problems to be faced in quantification must not be minimized. The problems are considerable and each adverse impact has its own unique set. Some of the problems are outlined in the paper. Unfortunately time is not on our side. Every year sees a rebirth of the concept of advertising from space in one form or another. Sooner or later, unless we are supremely vigilant, a proposal will be accepted and the consequences will be disastrous for our science. Hence the thrust of the EC Resolution before this General Assembly.

There is now great investment in astronomy as a science. That investment has produced some of the most exciting astronomy in all history. One only has to think of the spectacular discoveries of the HST, the advance in basic astrometric data represented by HIPPARCOS, and the unparalleled spectroscopic data base from IUE. We are currently looking forward to the fruits of the $10 \mathrm{~m}$ generation optical telescopes and sustained adventures in radio astronomy. Yet that tantalisingly brilliant future is at risk.

Unfortunately the interests of astronomy are now in conflict with other human activities. Astronomy has retreated to its mountains, deserts and into space. It can no longer retreat-there is nowhere left to go. The other competing interests are well heeled profitable and will claim enormous benefits for society. Some of the benefits are real but not all. We as astronomers will have to come to terms with the rest of society- but by the same token, the rest of society will have to come to terms with us - if astronomy is to survive as a major science. It would be a great disaster for the whole of science were the science of astronomy to be curtailed by the pollution produced terrestrially. Loss of investment would be disaster, loss of cosmic perspective would be a catastrophe.

\title{
2. IMPACT ON RADIO ASTRONOMY
}

\section{Morimoto (Space Science Course, Kagoshima University)}

The radio sky has continuously supplied "exotic objects" to astronomy, such as quasars, pulsars etc. After a while these objects proved to be important constituents of the Universe and provided a new look of the Universe.

Cosmic Background Radiation is the remnant of the blackbody radiation of Universe when it was cooled to about $3000 \mathrm{k}$. Density of quasars is higher in the distant part of the Universe and proves the Universe was more crowded in the early stage.

Spectral line emission from molecules formed in the interstellar gas clouds shows processes of star formation in the Galaxy and galaxies.

Technology in radio telescope in also continuously in a rapid progress. Recent success of HALUCA satellite and VSOP has opened a new era of high resolution radio imaging through space VLBI. Accuracy of source position measurements is approaching 1 microarcsec as level and trigonometry will soon cover whole Galaxy and beyond.

These are only a small portion of achievements of radio astronomy, but prove that it continucs to contribute important parts of the human knowledge of the Universe.

On the other hand, this exciting field of science is critically endangered by the more and more: intense usage of radio spectrum for communication and other purposes.

Frequencies below $\sim 300 \mathrm{MHz}$ (m-waves and longer)

Low frequency radio emission represents low density plasmas in Universe, which is clifficult to observe by other means. Examples are outer corona of the sun, envelope of the Galaxy and 
galaxies etc. Developments in array technology, image processing etc has enabled more sophisticated observations especially with higher angular resolution.

However, the situation may NOT allow the human being to enjoy this improvement fully. Protected bands are very narrow here, because this is the most crowded frequency band and allocation is given in terms of very small widths.

High resolution observations will become possible by use of arrays of space baselines. A different set of protection criteria will become necessary.

\section{0-30000 $\mathrm{MHz}$ (dm to $\mathrm{cm}$ waves)}

This is the band where most of the important discoveries were done and developments in new technology are in progress, but on the other hand, the most intensively used band by communications and other purposes. New demands such as satellite systems for personal pocket phones etc are rapidly growing but there is no resources of unused bands.

Situation will become more serious as more and more new demands will require space to earth transmission with relatively high power and to cover essentially whole surface to the earth. If some substantial measure NOT taken, human eyes in this important spectrum will be black out.

Above $30 \mathrm{GHz}$ ( $\mathrm{mm}$ and sub-mm waves)

Molecules in the interstellar clouds display a very large number of spectral lines mainly through rotational transition. It provides an unique possibility of direct observations of cool gas phase in Universe. Interference situation is not YET serious.

The band is not used very actively because of the cost and technical demands will be pointed to this area very soon.

\section{Frequency as the treasure of Nature}

Originally, communications lived in a small area, perhaps below $50 \mathrm{MHz}$. After the 2nd World War, the "big march" to the higher frequencies started and conquered the whole microwave region, which was, although sparsely, already inhabited by radio astronomy. Conquers allowed the original inhabitant to survive within narrow protected areas.

Frequency is like land. It is finite and easy to use up. It belongs to Nature. It is NOT owned by anyone.

Developments in the technology is at present encourage demands to USE the new frequency bands rather than protecting it, or in other words, Nature is protected only be the technical difficulties to use the band rather than the human wisdom.

\section{Conclusion}

We are about to lose the radio sky, which has continuously provided fascinations to the human being. Just like in other frontiers of the conservation of Nature, a use of the human wisdom to protect Nature rather than destroying is only the solution out or it.

\section{LIGHT POLLUTION: ITS DAMAGE TO EDUCATION AND CULTURE}

\section{J. R. Percy (Erindale Campus, University of Toronto)}

One could argue that light pollution has no impact on education or culture, because so few people care about real science. Perhaps people prefer bright lights, and "space art". The word education, however, comes from the Latin word to lead, and culture obviously refers to cultivation. It is our responsibility to educate the public in astronomy, and in the issues related to light pollution and related topics, and to cultive their appreciation of the night sky, and the universe.

Astronomy is important to education and culture because of its deep historical roots, its practical and philosophical applications, its aesthetic and emotional appeal (the beauty of the universe, and the sense of shared exploration and discovery), and its message about our place in time and space, and our cosmic roots and environment. In the classroom, it demonstrates the observational approach to the scientific method, and is a wonderful tool for teaching concepts of light, and gravity. It attracts young people to science and technology, and promotes public interest in science. Like all science, however, it is best taught through inquiry - simple, "hands-on" (or "eyes-on") activities. This is difficult in light-polluted skies.

Ironically, light pollution provides interesting opportunities for student projects, because it has scientific, technological, and societal aspects. Several groups have developed such projects, including co-operative, Internet-based projects in which students measure light pollution with simple devices, 